\title{
Efficacy, safety and pharmacokinetics of simeprevir and TMC647055/ritonavir with or without ribavirin and JNJ-56914845 in HCV genotype 1 infection
}

Stefan Bourgeois ${ }^{1 *}$, Hans Van Vlierberghe ${ }^{2}$, Christophe Moreno ${ }^{3}$, Hans Orlent $^{4}$, Frederik Nevens ${ }^{5}$, Keikawus Arastéh ${ }^{6}$, Yves Horsmans ${ }^{7}$, Jörn M. Schattenberg ${ }^{8}$, Peter Buggisch ${ }^{9}$, Sven Francque ${ }^{10}$, Leen Vijgen ${ }^{11}$, Thomas N. Kakuda ${ }^{12}$, Eva Hoeben ${ }^{13}$, Donghan Luo ${ }^{14}$, An Vandebosch ${ }^{13}$, Bert Jacquemyn ${ }^{11}$, Pieter Van Remoortere ${ }^{14}$ and René Verloes ${ }^{13}$

\begin{abstract}
Background: A Phase 2a, open-label study (NCT01724086) was conducted to assess the efficacy and safety of a once-daily, 2-direct-acting-antiviral-agent (2-DAA) combination of simeprevir + TMC647055/ritonavir \pm ribavirin and of the 3-DAA combination of simeprevir + TMC647055/ritonavir + JNJ-56914845 in chronic hepatitis C virus genotype (GT)1-infected treatment-naive and prior-relapse patients.

Methods: The study comprised four 12-week treatment panels: Panel 1 ( $n=10$; GT1a) and Panel 2-Arm 1 ( $n=12$; GT1b): simeprevir 75 mg once daily + TMC647055 450 mg once daily/ritonavir 30 mg once daily + ribavirin 1000-1200 mg/day; Panel 2-Arm 2 ( $n$ =9; GT1b): simeprevir 75 mg + TMC647055 450 mg/ritonavir 30 mg without ribavirin; Panel 3: simeprevir $75 \mathrm{mg}$ + TMC647055 600 mg/ritonavir 50 mg with (Arm 1: GT1a; $n=7$ ) or without (Arm 2: GT1b; $n=8$ ) ribavirin; Panel 4: simeprevir 75 mg + TMC647055 450 mg/ritonavir 30 mg + JNJ-56914845 30 mg once daily (Arm 1: n=22; GT1a/GT1b) or 60 mg once daily (Arm 2: $n=22 ; G T 1 a / G T 1 b)$. Primary endpoint was sustained virologic response 12 weeks after end of treatment (12 weeks of combination treatment; SVR12).
\end{abstract}

Results: In Panel 1 and Panel 2-Arm 1, 5/10 and 6/12 (50\%) GT1a/GT1b + ribavirin patients achieved SVR12, versus 3/9 (33\%) GT1b without ribavirin patients in Panel 2-Arm 2. In Panel 3-Arm 1 and Panel 3-Arm 2, 6/7 (86\%) GT1a + ribavirin and 4/8 (50\%) GT1b without ribavirin patients, respectively, achieved SVR12. In Panel 4, 10/14 (71\%) and 14/15 (93\%) GT1a patients in Arms 1 and 2 achieved SVR12 compared with 8/8 and 7/7 (100\%) GT1b patients in each arm, respectively. No deaths, serious adverse events (AEs), Grade 4 AEs or AEs leading to treatment discontinuation occurred.

Conclusions: The 2- and 3-DAA combinations were well tolerated. High SVR rates of 93\% and 100\% in GT1a- and GT1binfected patients, respectively, were achieved in this study by combining simeprevir with JNJ-56914845 $60 \mathrm{mg}$ and TMC647055/ritonavir.

Trial registration: NCT01724086 (date of registration: September 26, 2012)

Keywords: Simeprevir, TMC647055/ritonavir, JNJ-56914845, Ribavirin, Direct-acting antiviral agents, Hepatitis C virus, genotype 1, Efficacy, Safety

\footnotetext{
*Correspondence: stefan.bourgeois@zna.be

'Department of Gastroenterology \& Hepatology, ZNA Antwerp, Antwerpen,

Belgium

Full list of author information is available at the end of the article
} 


\section{Background}

Interferon (IFN)-free regimens comprising direct-acting antivirals (DAA) with different mechanisms of action can result in high sustained virologic response (SVR) rates in patients chronically infected with hepatitis $\mathrm{C}$ virus $(\mathrm{HCV})$. Combination regimens comprising an $\mathrm{HCV}$ NS3/4A protease inhibitor (PI), a non-nucleoside inhibitor (NNI) of the HCV NS5B polymerase and/or an HCV NS5A replication complex inhibitor with/without ribavirin have been shown to be successful in the treatment of chronic HCV infection [1]. For example, the combination of the PI paritaprevir, the NNI dasabuvir and the NS5A inhibitor ombitasvir, with ritonavir included as a pharmacologic booster for paritaprevir, is approved for the treatment of chronic HCV genotype (GT) 1 infection. In the PEARL-III (HCV GT1b) and PEARLIV (HCV GT1a) Phase 3 studies, treatment of previously untreated patients with paritaprevir/ritonavir, dasabuvir and ombitasvir for 12 weeks resulted in SVR 12 weeks after end of treatment (SVR12) rates of $99.5 \%$ and $97.0 \%$ in the presence of ribavirin, and $99.0 \%$ and $90.2 \%$ in the absence of ribavirin, respectively [2].

Simeprevir is a once-daily, HCV NS3/4A PI approved as part of an IFN-free combination with sofosbuvir for HCV GT1 infection. In addition, simeprevir with sofosbuvir is approved for HCV GT4 infection and $\mathrm{HCV} /$ human immunodeficiency virus (HIV) co-infection in the European Union (EU). Simeprevir is also approved in combination with pegylated IFN (pegIFN)/ribavirin for chronic HCV GT1 and GT4 infection in the United States and EU [3]. Simeprevir is a cytochrome P450 (CYP) 3A substrate and mild inhibitor (intestinal only). Simeprevir also inhibits organic anion transporting polypeptide (OATP) 1B1/3 and P-glycoprotein [4].

TMC647055 is an NNI of the HCV NS5B polymerase, binding in the NNI-1 pocket on the polymerase. TMC647055 has in vitro antiviral activity against HCV GT1, 3, 4, 5 and 6 [5]. In a Phase 1 study (NCT01202825), TMC647055 1000 mg twice daily in combination with simeprevir $150 \mathrm{mg}$ once daily for 10 days demonstrated good antiviral activity and was well tolerated in HCV GT1a- and GT1b-infected patients [6]. However, the systemic exposure to both compounds decreased during treatment to levels potentially lower than required for complete viral suppression. Coadministering TMC647055 with the potent CYP3A4 inhibitor ritonavir was expected to increase exposure by overcoming CYP3A4 induction [5].

JNJ-56914845 (previously known as GSK-2336805) is a potent $\mathrm{HCV}$ NS5A replication complex inhibitor, with in vitro antiviral activity against $\mathrm{HCV}$ GT1, 4, 5 and $6 \mathrm{a} / 6 \mathrm{~b}[7,8]$. It is a CYP3A and P-glycoprotein substrate and, in vitro, it inhibits P-glycoprotein, OATP1B1/3 and breast cancer resistance protein (data on file).
This Phase 2a, open-label study (NCT01724086) assessed the efficacy, pharmacokinetics, safety and tolerability of the 2-DAA combination of simeprevir with TMC647055 and a low dose of ritonavir with or without ribavirin for 12 weeks in chronic HCV GT1-infected treatment-naïve and prior-relapse patients. The study also included the addition of a third DAA, JNJ-56914845, to the simeprevir and TMC647055 combination to assess the potential of further increasing the efficacy of the regimen by combining three DAAs with different mechanisms of action. Results from the final analysis, when all patients in all panels had completed the study, are presented here.

\section{Methods}

\section{Patients and study design}

This was a Phase 2a, open-label study, conducted between 12 September 2012 and 16 December 2014 at 12 sites in Belgium and Germany. The study was approved by the Institutional Review Board or Independent Ethics Committee at each participating centre, and met the ethical principles of the Declaration of Helsinki and Good Clinical Practice guidelines. All patients provided written, informed consent.

Adults (18-70 years of age) with chronic HCV GT1a/ GT1b infection and screening plasma HCV RNA $>10,000 \mathrm{IU} / \mathrm{mL}$ who were treatment-naïve or had relapsed following previous treatment with pegIFN/ribavirin were eligible for inclusion. Patients had to have a documented liver biopsy within 3 years of the screening visit, or have an elastography prior to first dosing.

Exclusion criteria included liver cirrhosis, hepatic decompensation, liver disease of non-HCV aetiology, infection/co-infection with non-GT1a/GT1b HCV, hepatitis $A$ or $B$, or $\mathrm{HIV}-1 /-2$, and significant laboratory abnormalities, including total bilirubin $\geq 1.5 \mathrm{x}$ upper limit of normal and platelet count $<90,000 / \mathrm{mm}^{3}$. Patients who had received prior $\mathrm{HCV}$-specific DAA treatment or a liver transplant were also excluded.

The study comprised four 12-week treatment panels (Fig. 1). Panels 1 and 2, which ran in parallel, comprised the first part of the study and were sequentially followed by Panels 3 and 4. Panels $1-3$ evaluated simeprevir in combination with TMC647055/ritonavir (at two different doses of TMC647055) with ribavirin in GT1a-infected patients and with or without ribavirin in GT1b-infected patients. Panel 4 evaluated the triple combination of simeprevir with TMC647055/ritonavir plus the NS5A inhibitor JNJ-56914845 at a low and high dose in GT1a/b-infected patients.

More specifically, patients in Panels 1 and 2 received simeprevir $75 \mathrm{mg}$ once daily, TMC647055 $450 \mathrm{mg}$ once daily, ritonavir $30 \mathrm{mg}$ once daily and ribavirin 10001200 mg (patients in Panel 2-Arm 2 did not receive ribavirin). Patients in Panel 3 received simeprevir $75 \mathrm{mg}$ 


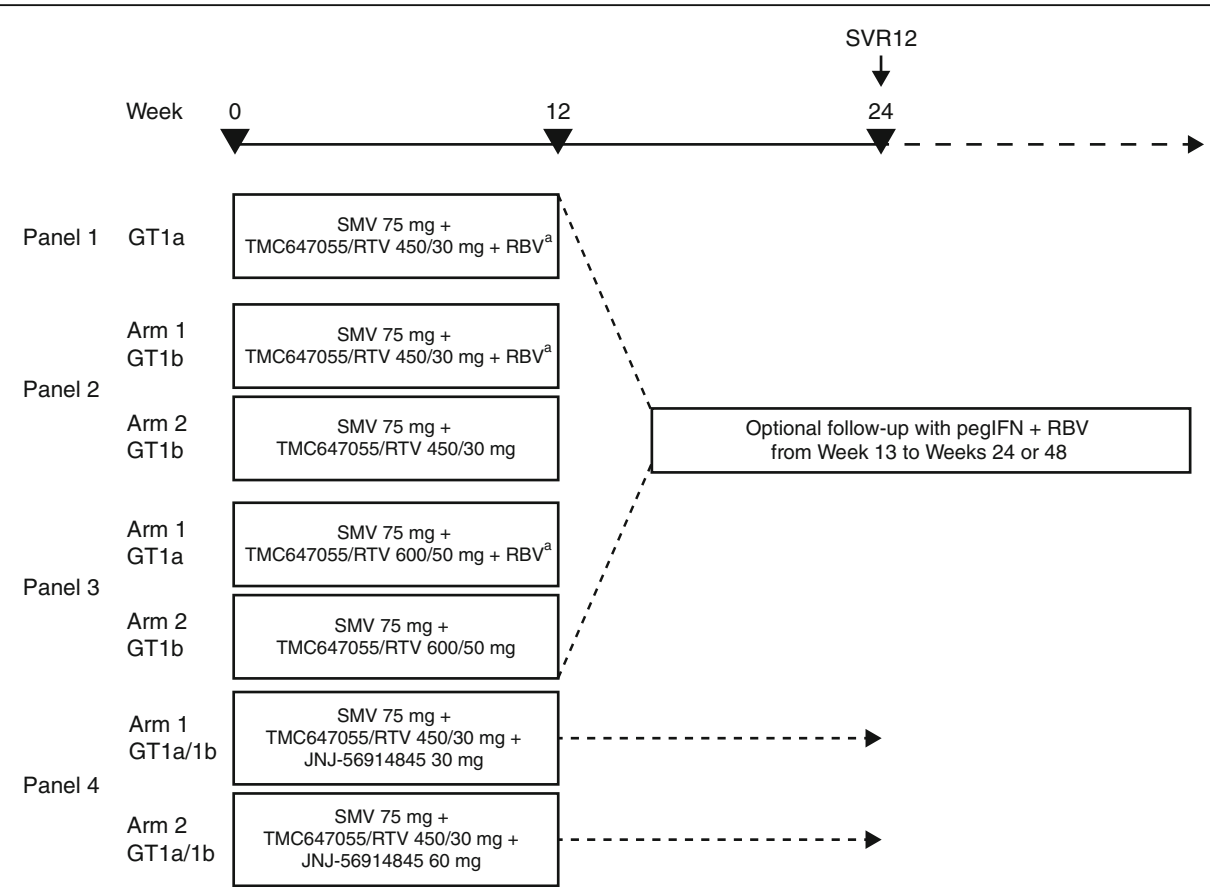

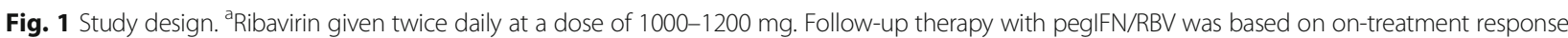
and was initiated only if: Week $4 \mathrm{HCV}$ RNA $\geq 25 \mathrm{IU} / \mathrm{mL}$ (Panels 1-3: 36 weeks of follow-up therapy); Week $4 \mathrm{HCV}$ RNA $<25 \mathrm{IU} / \mathrm{mL}$ detectable or HCV RNA confirmed detectable between Week 4 and Week 11 (Panels 1-2: 12 weeks of follow-up therapy). GT genotype, HCV hepatitis C virus, pegIFN pegylated interferon a-2a, RBV ribavirin, RTV ritonavir, SMV simeprevir, SVR12 sustained virologic response 12 weeks after end of treatment

once daily, TMC647055 at a higher dose of $600 \mathrm{mg}$ once daily, ritonavir $50 \mathrm{mg}$ once daily and ribavirin 1000 $1200 \mathrm{mg}$ (patients in Panel 3-Arm 2 did not receive ribavirin). Patients in Panel 4 received simeprevir $75 \mathrm{mg}$ once daily, TMC647055 $450 \mathrm{mg}$ once daily, ritonavir $30 \mathrm{mg}$ once daily and JNJ-56914845 30 (Arm 1) or $60 \mathrm{mg}$ (Arm 2) once daily. Simeprevir was dosed at $75 \mathrm{mg}$ once daily (rather than the $150 \mathrm{mg}$ dose approved) to account for CYP3A inhibition by ritonavir, which is not a part of the approved regimen for simeprevir. Further details on the dosing rationale are provided in Additional file 1. For ethical reasons, patients in Panels 1-3 received follow-up treatment with pegIFN/ribavirin for an additional 12 or 36 weeks if their Week-4 HCV RNA levels did not comply with predefined criteria (Fig. 1). For patients in Panel 4 receiving the 3-DAA regimen, no follow-up treatment was planned.

Central randomisation was implemented in Panel 2, with patients randomly assigned 1:1 to Arm 1 or Arm 2 based on a computer-generated schedule prepared before the study, balanced by using randomly permuted blocks, and stratified by IL28B genotype (CC and nonCC). Patients in Panel 3 were not randomised as their treatment was dependent on their $\mathrm{HCV}$ geno/subtype (1a or 1b) (Fig. 1). In Panel 4, patients were randomised in a 1:1 ratio between Arm 1 or Arm 2, stratified by $\mathrm{HCV}$ geno/subtype.
To avoid unnecessary drug exposure, the following virologic stopping rules were adopted: all study drugs were discontinued for patients with viral breakthrough (confirmed on-treatment increase of $>1 \log _{10} \mathrm{IU} / \mathrm{mL}$ in $\mathrm{HCV}$ RNA from the lowest level reached, or confirmed $\mathrm{HCV}$ RNA $>100 \mathrm{IU} / \mathrm{mL}$ in patients whose HCV RNA had previously been $<25 \mathrm{IU} / \mathrm{mL}$ ), or with inadequate virologic response (confirmed HCV RNA $>100 \mathrm{IU} / \mathrm{mL}$ at Week 4 or afterwards until Week 11).

Details on protocol deviations are provided in Additional file 1.

\section{Outcomes}

The primary efficacy endpoint was SVR12. Patients achieved SVR12 if they received no pegIFN/ribavirin follow-up treatment after 12 weeks of combination treatment (Panels 1-3 only) and achieved HCV RNA $<25 \mathrm{IU} / \mathrm{mL}$ undetectable/detectable 12 weeks after actual end of treatment (Panels 1-4). Patients in Panels 1-3 who received follow-up therapy and had HCV RNA $<25 \mathrm{IU} / \mathrm{mL}$ at 12 weeks after end of treatment were also classed as having achieved SVR12; however, they were considered as failures with regards to the primary endpoint of the study, which focused on the 12-week DAA therapy. The primary safety endpoints included the proportion of patients with adverse events (AEs), serious AEs (SAEs) or abnormal changes in safety-related 
laboratory values. Secondary endpoints included: the proportion of patients with SVR 24 weeks after end of treatment (SVR24); Week-4 pharmacokinetics of the study drugs; on-treatment virologic failure, including patients with viral breakthrough (defined in the previous section); viral relapse (defined as HCV RNA $<25 \mathrm{IU} / \mathrm{mL}$ undetectable at the actual end of treatment and confirmed HCV RNA $\geq 25 \mathrm{IU} / \mathrm{mL}$ during post-treatment follow-up); and the presence of $\mathrm{HCV} \mathrm{NS3/4A,} \mathrm{NS5A}$ and/or NS5B variants at baseline and at time of failure in patients not achieving SVR.

\section{Assessments}

Blood samples for HCV RNA level determination were collected at screening and at predefined time points throughout the treatment phase and follow-up period. HCV RNA was measured using the COBAS ${ }^{\circ}$ TaqMan $^{\circ}$ HCV Test version 2.0 (Roche Molecular Diagnostics, Pleasanton, CA, USA) for use with the High Pure System assay (lower limit of quantification: $25 \mathrm{IU} / \mathrm{mL}$; limit of detection: $10-15 \mathrm{IU} / \mathrm{mL}$ ).

$\mathrm{HCV}$ geno/subtypes were determined pre-treatment based on sequencing of a part of the NS5B gene (at baseline) when available or by the VERSANT ${ }^{\circ} \mathrm{HCV}_{\text {Genotype }}$ 2.0 assay (LiPA) or Trugene HCV Genotyping assay (at screening) (Siemens Healthcare Diagnostics, Erlangen, Germany).

Standard population-based sequencing of the $\mathrm{HCV}$ NS3/4A and NS5B regions in Panels $1-3$ and of the NS3/4A, NS5A and NS5B regions in Panel 4 was performed at baseline for all patients and post-baseline for patients not achieving SVR12 based on the HCV RNA changes observed in each individual patient and the limits of the sequencing assay.

Plasma pharmacokinetic samples for simeprevir, TMC647055, ritonavir and JNJ-56914845 were collected over $24 \mathrm{~h}$ (pre-dose, 1, 2, 3, 4, 5, 6, 8, 10, 12 and $24 \mathrm{~h}$ post-dose) at Week 4 from all patients and assayed using validated liquid chromatography-tandem mass spectrometry methods with lower limits of quantification for simeprevir, TMC647055, ritonavir and JNJ-56914845 of 5.0, 5.0, 2.0 and $1.0 \mathrm{ng} / \mathrm{mL}$, respectively (data on file) [9]. Pharmacokinetic parameters including maximum plasma concentration $\left(\mathrm{C}_{\max }\right)$ and area under the plasma concentration-time curve over $24 \mathrm{~h}\left(\mathrm{AUC}_{0-24 \mathrm{~h}}\right)$ were calculated using noncompartmental analysis (Phoenix WinNonlin ${ }^{\circ}$ 6.2.1; Certara, Princeton, NJ, USA).

AEs were monitored throughout the treatment phase and follow-up period and up to 24 weeks after actual end of treatment. AEs were coded using the Medical Dictionary for Regulatory Activities (version 16.0 for Panels 1 and 2 and version 17.0 for Panels 3 and 4).

\section{Statistical analyses}

Statistical analyses were performed using $\mathrm{SAS}^{\circ}$ version 9.1 or higher (SAS Institute Inc, Cary, NC, USA).

No formal sample size calculations were performed as this was a proof-of-concept study. For each efficacy endpoint, the proportion of patients was summarised descriptively together with a $95 \%$ confidence interval by treatment group and population. The change in $\log _{10}$ HCV RNA from baseline at all time points was calculated using descriptive statistics. Pharmacokinetic parameters were calculated using non-compartmental analysis (Phoenix WinNonlin ${ }^{\circ}$ 6.2.1). All safety and tolerability data were summarised descriptively.

All analyses were conducted using data from the intent-to-treat population, which comprised all patients who received at least one dose of study drug.

\section{Results}

\section{Patient disposition and baseline characteristics}

In total, 125 patients were screened, and $90(n=10,21,15$ and 44 in Panels 1, 2, 3 and 4, respectively) were treated (intent-to-treat population) (Fig. 2). Four (3\%) patients discontinued the study prematurely (lost to follow-up, $n=3$ [Panels 2 and 3]; withdrawal of consent, $n=1$ [Panel 4]).

In Panel 1, 5/10 (50\%) patients completed their treatment regimen. In Panel 2, the majority of patients completed treatment (GT1b/with ribavirin group: 11/12 [92\%]; GT1b/without ribavirin group, 8/9 [89\%]). Most patients also completed treatment in Panel 3 (GT1a/with ribavirin, 7/7 [100\%]; GT1b/without ribavirin, 7/8 [88\%]) and Panel 4 (30-mg group, 22/22 [100\%]; 60-mg group, 21/22 [96\%]). All treatment discontinuations were due to viral breakthrough, as detailed in the next section.

Baseline demographics and disease characteristics are shown in Table 1.

\section{Virologic response}

Figure 3 shows the treatment outcome in each of the four panels. In Panel 1, 5/10 (50\%) patients achieved SVR12. In Panel 2, SVR12 was achieved in 6/12 (50\%) and $3 / 9(33 \%)$ patients in the GT1b/with ribavirin and GT1b/without ribavirin groups, respectively. In Panel 3, $6 / 7(86 \%)$ and $4 / 8(50 \%)$ patients in the GT1a/with ribavirin and GT1b/without ribavirin groups, respectively, achieved SVR12. In Panel 4, SVR12 was achieved in $18 / 22(82 \%)$ and $21 / 22(95 \%)$ patients in the JNJ-56914845 30- and 60-mg groups, respectively. In the 30-mg group, SVR12 was achieved in 10/14 (71\%) GT1a/other-infected patients and in all $(8 / 8 ; 100 \%)$ GT1b-infected patients. In the 60-mg group, SVR12 was achieved in 14/15 (93\%) GT1a/other-infected patients and in all $(7 / 7 ; 100 \%)$ GT1b-infected patients. 


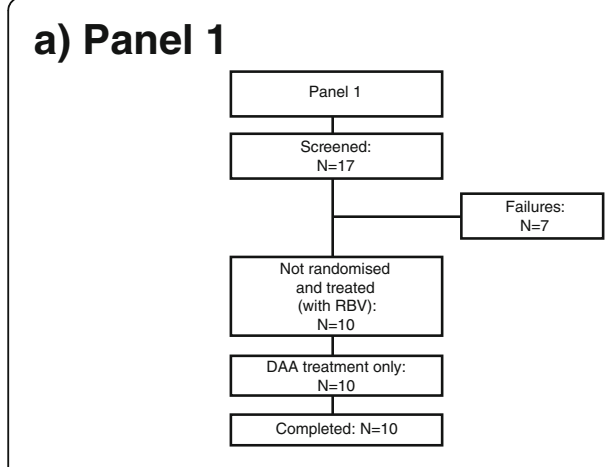

b) Panel 2

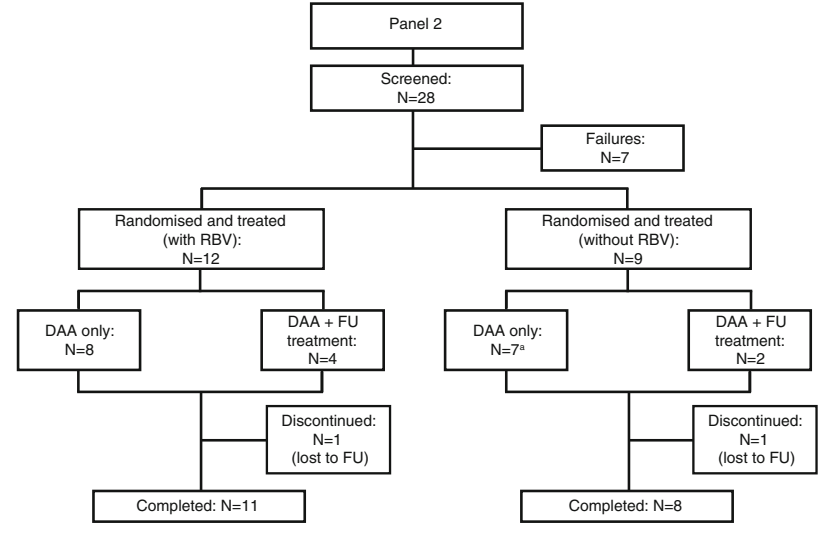

\section{c) Panel 3}
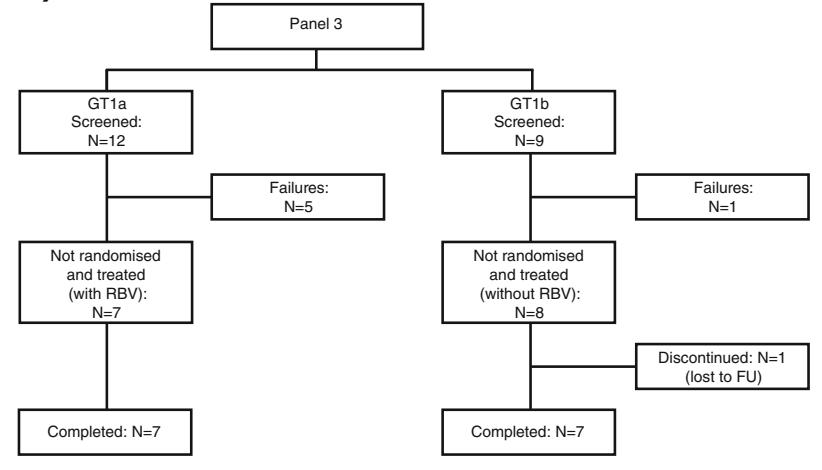

\section{d) Panel 4}

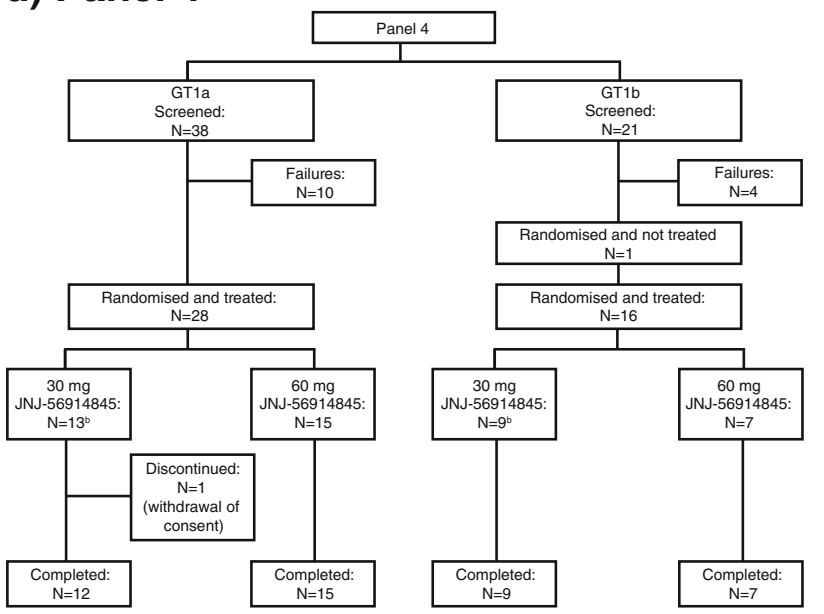

Fig. 2 Patient disposition. In Panel 2, one patient in the GT1b/without RBV group had $<25 \mathrm{IU} / \mathrm{mL}$ detectable HCV RNA at Week 4 and, therefore, met

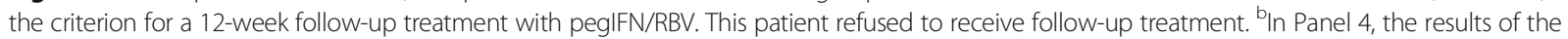
Trugene or LiPA test performed at screening were used to randomise the patients to the 30-mg or 60-mg dose of JNJ-56914845. For analyses purposes, NS5B sequencing was used to determine the HCV geno/subtype. Based on this method, the geno/subtype for two patients in the 30-mg group was found to be non-1a, non-1b (1c, $n=1 ; 11, n=1)$ based on geno/subtyping using NS5B sequencing. These two patients were analysed together with the GT1a-infected patients in the category 'GT1a/other'. DAA direct-acting antiviral agent, FU follow-up, GT genotype, HCV hepatitis C virus, pegIFN pegylated interferon a-2a, RBV ribavirin

All patients who achieved SVR12 also went on to achieve SVR24, with the exception of one patient in Panel 1 and one patient in the GT1b/without ribavirin group in Panel 3 who had HCV RNA $\geq 25 \mathrm{IU} / \mathrm{mL}$ 24 weeks after end of treatment $(1,830,000 \mathrm{IU} / \mathrm{mL}$ and $2770 \mathrm{IU} / \mathrm{mL}$, respectively).

An overview of treatment outcome, including reasons for not achieving SVR12, is shown in Fig. 3. A total of 5/10 (50\%), $1 / 12(8 \%)$ and $1 / 9(11 \%)$ patients in Panel 1 and in the GT1b/with ribavirin and GT1b/without ribavirin groups of Panel 2, respectively, experienced viral breakthrough. In Panel 3, 1/8 (13\%) patients in the GT1b/without ribavirin group had viral breakthrough compared with $1 / 22(5 \%)$ patients in the $60-\mathrm{mg}$ group of Panel 4 (Fig. 3). Among the patients with undetectable HCV RNA at end of treatment and with at least one post-treatment follow-up HCV RNA measurement available, $2 / 6$ patients (33\%) in each of the Panel 2 and Panel 3 GT1b/without ribavirin groups and 4/14 (18\%) GT1a-infected patients in the Panel 4 30mg group experienced viral relapse. At the time of the final analysis, one patient in Panel 1 and one patient in the Panel 3 GT1b/without ribavirin group achieved SVR12 but had HCV RNA $\geq 25 \mathrm{IU} / \mathrm{mL} 24$ weeks after end of treatment.

\section{Virologic resistance testing}

At baseline, the NS3 Q80K polymorphism was observed in 2/10 (20\%) GT1a-infected patients in Panel 1 and in 2/13 (15\%) GT1a-infected patients in the 30-mg group 


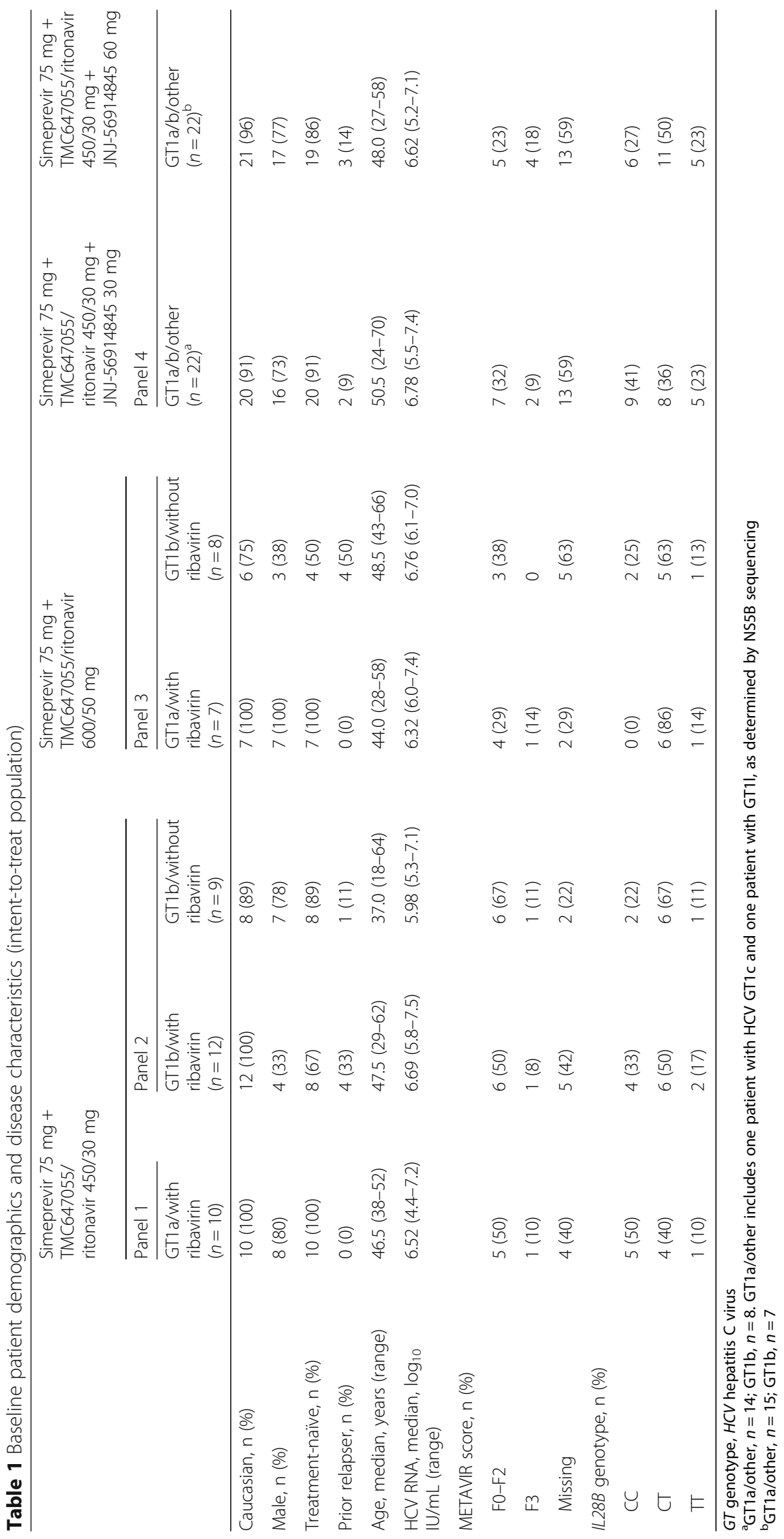




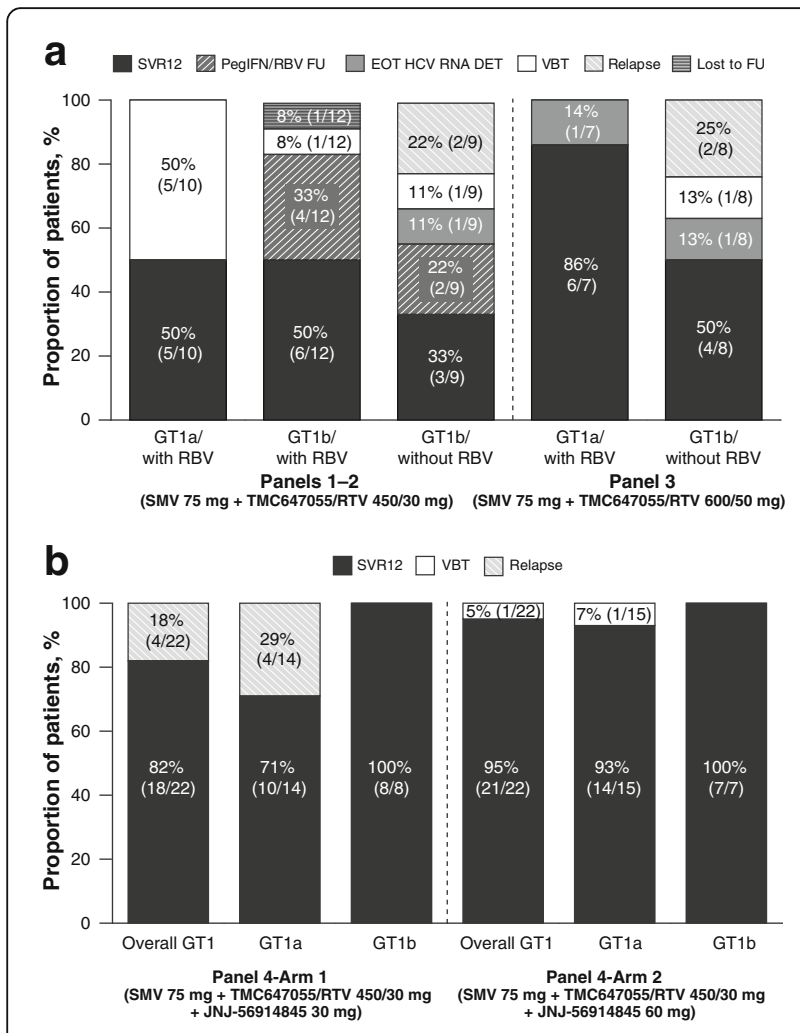

Fig. 3 Treatment outcome in (a) Panels 1-3, and (b) Panel 4. SVR12 data shown represent SVR12 achieved after treatment with only the combination of SMV + TMC647055/RTV \pm RBV. In the Panel 2 GT1b/with RBV and GT1b/without RBV arms, four and two patients, respectively, had follow-up therapy with pegIFN/RBV and are, therefore, not included in the SVR12 category that focuses on SVR12 after 12 weeks of DAA treatment. Among them, five patients achieved SVR12 after completing follow-up therapy; one patient refused to receive follow-up treatment and was lost to follow-up. One additional patient in the Panel $2 \mathrm{GT1b} /$ without RBV group had $<25 \mathrm{IU} / \mathrm{mL}$ detectable HCV RNA at Week 4 and, therefore, met the criterion for the 12-week follow-up treatment with pegIFN/RBV; however, this patient refused to receive followup treatment. DAA direct-acting antiviral agent, DET detectable, EOT end of treatment, FU follow-up, GT genotype, HCV hepatitis $C$ virus, pegIFN pegylated interferon a-2a, RBV ribavirin, RTV ritonavir, SMV simeprevir, SVR12 sustained virologic response 12 weeks after end of treatment, VBT viral breakthrough

in Panel 4. Neither of the two GT1a-infected patients with baseline Q80K in Panel 1, and one of the two patients in Panel 4 achieved SVR12. In 5/42 (12\%) patients with NS5A sequencing data available in Panel 4, baseline NS5A polymorphisms were detected at positions 28, 30, 31 and/or 93. Of those, only L31M $(n=1)$ and Y93C (in combination with $\mathrm{M} 28 \mathrm{~V} ; n=1$ ), both detected in GT1a-infected patients, are associated with in vitro resistance to JNJ-56914845 [8]. Details on NS5B baseline polymorphisms are provided in Additional file 1.

Virologic failure (including the two patients who achieved SVR12 but had HCV RNA $\geq 25 \mathrm{IU} / \mathrm{mL} 24$ weeks after end of treatment) was, in the majority of patients, associated with the emergence of simeprevir and TMC647055 resistance-associated variants (RAVs) in Panels 1-3 and simeprevir and JNJ-56914845 RAVs with or without TMC647055 RAVs in Panel 4 at time of failure. In line with the previously characterised simeprevir resistance profile, most GT1b-infected patients harboured an emerging mutation at NS3 position 168, while emerging mutations in GT1a-infected patients were mostly observed at NS3 position 155 (R155K alone or in combination with a Q80R; R155S) at time of failure. TMC647055 RAVs were only observed at NS5B position 495 (mainly P495L), consistent with the resistance profile of NNI-1 polymerase inhibitors. In all patients with virologic failure in Panel 4, emerging JNJ-56914845 RAVs at NS5A positions 30 and/or 31 were detected at time of failure. Treatment-emergent RAVs in NS3 and NS5B became undetectable in many of the patients after treatment was stopped, while emerging RAVs in NS5A could still be detected by population sequencing at the end of the study in the five patients who failed the 3-DAA treatment.

\section{Pharmacokinetics}

Co-administration of high-dose TMC647055/ritonavir $(600 / 50 \mathrm{mg})$ increased simeprevir exposure compared with low-dose TMC647055/ritonavir (450/30 mg). Simeprevir $\mathrm{C}_{\max }$ values were increased by 2.0 -fold and $\mathrm{AUC}_{0-24 \mathrm{~h}}$ values by 1.9 - to 2.3 -fold, respectively, following co-administration with TMC647055/ritonavir (600/50 mg vs 450/30 mg) with ribavirin (Panel 3-Arm 1 vs Panel 1) or without ribavirin (Panel 3-Arm 2 vs Panel 2-Arm 2) (Additional file 2: Figure S1a).

A more than dose-proportional increase in TMC647055 exposure occurred following co-administration of TMC647055/ ritonavir $600 \mathrm{mg} / 50 \mathrm{mg}$ once daily (Panel 3-Arm 2) compared with $450 \mathrm{mg} / 30 \mathrm{mg}$ once daily (Panel 2-Arm 2) with simeprevir $\left(\mathrm{C}_{\text {max }}\right.$ : 2.6-fold increase; $\mathrm{AUC}_{0-24 \mathrm{~h}}$ : 2.4-fold increase) (Additional file 2: Figure S1b).

Co-administration of high-dose JNJ-56914845 $60 \mathrm{mg}$ once daily with simeprevir $75 \mathrm{mg}$ once daily + TMC647055/ritonavir $450 \mathrm{mg} / 30 \mathrm{mg}$ once daily increased simeprevir exposure (1.2-fold increase in both $\mathrm{C}_{\max }$ and $\mathrm{AUC}_{0-24 \mathrm{~h}}$ vs without JNJ-56914845) (Additional file 2: Figure S1a), but had minimal effect on TMC647055 and ritonavir exposure (Additional file 2: Figure S1b and c).

A dose-proportional increase in exposure to JNJ$5691484560 \mathrm{mg}$ versus $30 \mathrm{mg}$ once daily was observed when co-administered with simeprevir $75 \mathrm{mg}+$ TMC647055/ritonavir $450 \mathrm{mg} / 30 \mathrm{mg}$ once daily (Panel 4-Arm 2 vs Panel 4-Arm 1: $\mathrm{C}_{\max } 821$ vs 389 ng/mL, $\mathrm{AUC}_{0-24 \mathrm{~h}} 7747$ vs $3358 \mathrm{ng} \cdot \mathrm{h} / \mathrm{mL}$ ) (Additional file 2: Figure S1d; Additional file 3: Table S1).

No differences were observed in simeprevir, TMC647055 or ritonavir pharmacokinetics based on ribavirin use 
(Additional file 4: Table S2, Additional file 5: Table S3 and Additional file 6: Table S4).

\section{Safety}

No patients discontinued any of the study drugs due to an $\mathrm{AE}$, and there were no SAEs or deaths.

The incidence of AEs was similar between Panels 1 and 2, and Panel 3 (Table 2). In Panels 1 and 2, all reported AEs were Grade 1 or 2, and there were no clinically relevant differences among patients treated with ribavirin and those who did not receive ribavirin in terms of the incidence or severity of AEs. In Panel 3, all AEs were Grade 1 or 2 , with the exception of one patient (GT1b/without ribavirin group) who experienced a Grade $3 \mathrm{AE}$ (hypercholesterolaemia). This AE was not serious and was not considered related to simeprevir, TMC647055 or ritonavir by the investigator; the AE was ongoing at the time of the final analysis. A higher incidence of patients in Panel 3 treated with ribavirin experienced a Grade 1 or $2 \mathrm{AE}$ compared with those who did not receive ribavirin. All AEs in Panel 4 were Grade 1 or 2, with the exception of one patient who experienced a Grade 3 increase in white blood cell count (not related to any study drug). There were no clinically relevant differences in the incidence of AEs between patients treated with JNJ-56914845 at either dose (Table 2).

The most frequently (in $>20 \%$ of patients) reported AEs during the treatment phase were as follows; Panels 1 and 2: headache (14/31 [45\%]), fatigue (9/31 [29\%]) and influenza-like illness (7/31 [23\%]); Panel 3: nausea (7/15 [47\%]), headache (7/15 [47\%]), diarrhoea (5/15
[33\%]), fatigue (4/15 [27\%]) and pruritus (4/15 [27\%]); Panel 4: headache (15/44 [33\%]), diarrhoea (14/44 [32\%]) and fatigue (13/44 [30\%]).

The majority of graded laboratory abnormalities were Grade 1 or 2 and were not clinically significant. Grade 3 or 4 laboratory abnormalities were observed in $<10 \%$ of patients in all panels (further details are provided in Additional file 1). Hyperbilirubinaemia was reported as a laboratory-related AE in 1 (3\%) patient in Panels 1 and 2 (considered possibly related to TMC647055 and simeprevir) and 1 (2\%) patient in the 60-mg group in Panel 4 (considered possibly related to TMC647055, ritonavir and JNJ-56914845, and probably related to simeprevir). Both hyperbilirubinaemia AEs were Grade 1 or 2 in severity.

\section{Discussion}

This was a proof-of-concept study performed to explore the efficacy and safety of the 2-DAA combination of the HCV NS3/4A PI simeprevir and the HCV NS5B NNI TMC647055 administered with ritonavir \pm ribavirin, and of the 3-DAA combination of simeprevir, TMC647055 and the HCV NS5A inhibitor JNJ-56914845 administered with ritonavir in HCV GT1-infected patients. The results suggest that the addition of JNJ-56914845 (60 mg) to the 2-DAA regimen of simeprevir and TMC647055 was highly beneficial in improving the efficacy of the combination treatment in patients with HCV GT1 infection.

Overall, the 2-DAA combination of low-dose TMC647055/ritonavir plus simeprevir, with or without ribavirin, did not demonstrate high efficacy in GT1-

Table 2 Summary of adverse events during the treatment phase (intent-to-treat population)

\begin{tabular}{|c|c|c|c|c|c|c|c|}
\hline & \multicolumn{3}{|c|}{$\begin{array}{l}\text { Simeprevir } 75 \text { mg + } \\
\text { TMC647055/ritonavir } \\
450 / 30 \text { mg }\end{array}$} & \multirow{2}{*}{\multicolumn{2}{|c|}{$\begin{array}{l}\text { Simeprevir } 75 \text { mg + } \\
\text { TMC647055/ritonavir } \\
600 / 50 \text { mg } \\
\text { Panel } 3 \\
\end{array}$}} & \multirow{3}{*}{$\begin{array}{l}\text { Simeprevir } 75 \text { mg + } \\
\text { TMC647055/ritonavir } \\
450 / 30 \text { mg }+ \\
\text { JNJ-56914845 } 30 \mathrm{mg} \\
\text { Panel } 4 \\
\text { GT1a/b/other } \\
(n=22)^{\mathrm{a}}\end{array}$} & \multirow{3}{*}{$\begin{array}{l}\text { Simeprevir } 75 \text { mg + } \\
\text { TMC647055/ritonavir } \\
450 / 30 \mathrm{mg}+\text { JNJ-56914845 } \\
60 \mathrm{mg} \\
\\
\begin{array}{l}\text { GT1a/b/other } \\
(n=22)^{\mathrm{b}}\end{array}\end{array}$} \\
\hline & \multirow{2}{*}{$\begin{array}{l}\text { Panel } 1 \\
\text { GT1a/with } \\
\text { ribavirin } \\
(n=10)\end{array}$} & \multicolumn{2}{|l|}{ Panel 2} & & & & \\
\hline & & $\begin{array}{l}\text { GT1b/with } \\
\text { ribavirin } \\
(n=12)\end{array}$ & $\begin{array}{l}\text { GT1b/without } \\
\text { ribavirin } \\
(n=9)\end{array}$ & $\begin{array}{l}\text { GT1a/with } \\
\text { ribavirin } \\
(n=7)\end{array}$ & $\begin{array}{l}\text { GT1b/without } \\
\text { ribavirin } \\
(n=8)\end{array}$ & & \\
\hline Any AE, n (\%) & $10(100)$ & $12(100)$ & 7 (78) & $6(86)$ & $6(75)$ & $20(91)$ & $22(100)$ \\
\hline Worst Grade 1, n (\%) & $6(60)$ & $7(58)$ & $4(44)$ & $5(71)$ & $3(38)$ & $15(68)$ & $15(68)$ \\
\hline Worst Grade 2, n (\%) & $4(40)$ & $5(42)$ & $3(33)$ & $1(14)$ & $2(25)$ & $5(23)$ & $6(27)$ \\
\hline Worst Grade 3, n (\%) & $0(0)$ & $0(0)$ & $0(0)$ & $0(0)$ & $1(13)^{c}$ & $0(0)$ & $1(5)^{d}$ \\
\hline Worst Grade 4, n (\%) & $0(0)$ & $0(0)$ & $0(0)$ & $0(0)$ & $0(0)$ & $0(0)$ & $0(0)$ \\
\hline $\begin{array}{l}\text { Leading to permanent stop of } \\
\text { study drugs, } n(\%)\end{array}$ & $0(0)$ & $0(0)$ & $0(0)$ & $0(0)$ & $0(0)$ & $0(0)$ & $0(0)$ \\
\hline Serious AE, n (\%) & $0(0)$ & $0(0)$ & $0(0)$ & $0(0)$ & $0(0)$ & $0(0)$ & $0(0)$ \\
\hline Death, n (\%) & $0(0)$ & $0(0)$ & $0(0)$ & $0(0)$ & $0(0)$ & $0(0)$ & $0(0)$ \\
\hline
\end{tabular}

$A E$ adverse event, $G T$ genotype

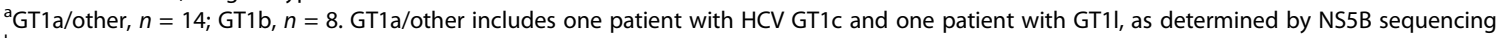

${ }^{\mathrm{b}} \mathrm{GT} 1 \mathrm{a} /$ other, $n=15 ; \mathrm{GT} 1 \mathrm{~b}, n=7$

'Hypercholesterolaemia, not related to any study drug

${ }^{d}$ Increase in white blood cell count, not related to any study drug 
infected patients (SVR12: 33\%-50\%). The SVR12 rate in HCV GT1a-infected patients treated with the 2-DAA combination and ribavirin was $86 \%(6 / 7)$ for the high dose of TMC647055/ritonavir, and 50\% (5/10) for the low dose of TMC647055/ritonavir, indicating a dosedependent increase in efficacy of TMC647055/ritonavir. The increased dose was not associated with an increase in AEs and there were no safety concerns regarding the use of ritonavir. Although the 2-DAA combination and the high dose of TMC647055/ritonavir demonstrated a higher SVR12 rate in GT1a-infected patients compared with the low dose of TMC647055/ritonavir, this was not observed in GT1b-infected patients who received the 2-DAA combination without ribavirin (SVR12 for the low- vs high-dose TMC647055/ritonavir: 33\% [3/9] vs $50 \%$ [4/8], respectively). Although data are limited, these suggest the addition of ribavirin may be required to increase SVR12 in a 2-DAA regimen combining an NS3/4A PI and an NS5B NNI.

In Panel 1, neither of the two GT1a-infected patients with an NS3 Q80K polymorphism at baseline achieved SVR12. Moreover, viral breakthrough was frequently observed with the 2-DAA combination (ranging from 8-50\%) compared with a frequency of $5 \%$ with the 3-DAA combination (1/22 patient who received the 3-DAA combination with JNJ-56914845 [60 mg]).

The 3-DAA combination resulted in an overall SVR12 rate of $95 \%$ with $60 \mathrm{mg}$ JNJ-56914845. All GT1binfected patients $(7 / 7 ; 100 \%)$ treated with the 3-DAA combination achieved SVR12, and the majority of GT1ainfected patients $(14 / 15$; 93\%) also achieved SVR12 following treatment with this regimen. The SVR12 rates observed with this combination therapy are consistent with those reported in Phase 3 studies that assessed the combination of the PI paritaprevir with the NNI dasabuvir and the NS5A inhibitor ombitasvir in treatmentnaïve or treatment-experienced, non-cirrhotic, $\mathrm{HCV}$ GT1-infected patients [2, 10-12].

The simeprevir resistance profile observed in all panels in this study was similar to that observed in the simeprevir Phase 3 studies in the presence of pegIFN/ribavirin [13-15]. A genotypic and phenotypic analysis of baseline $\mathrm{HCV}$ isolates from all patients who participated in this study, as well as of isolates obtained at the time of failure and at the end of study from patients with virologic failure, are described in full in a separate paper (Leen Vijgen, Kim Thys, An Vandebosch, Pieter Van Remoortere, René Verloes, Sandra De Meyer. Virology analysis in HCV genotype 1-infected patients treated with the combination of simeprevir and TMC647055/ritonavir, with and without ribavirin, and JNJ-56914845, in preparation).

The interaction between the drugs assessed in this study had previously been evaluated in healthy volunteers [16]. In that study, co-administration of simeprevir increased exposure $\left(\mathrm{AUC}_{0-24 \mathrm{~h}}\right)$ of JNJ-56914845 2.6-fold with no additional effect when TMC647055/ritonavir was added. Co-administration with JNJ-56914845 resulted in small increases in the plasma concentrations of simeprevir and TMC647055. These increases were not considered to be clinically relevant for either DAA [16]. In the current study, administration of simeprevir $75 \mathrm{mg}$ once daily in combination with TMC647055/ritonavir in HCV-infected patients resulted in simeprevir exposures within the range observed in historical data for simeprevir when dosed at $150 \mathrm{mg}$ once daily (data on file). In addition, high exposure to TMC647055 was achieved when administered in combination with ritonavir.

Both the 2-DAA and the 3-DAA regimens were well tolerated. There were no deaths, SAEs, Grade 4 AEs or AEs leading to temporary/permanent treatment discontinuation in any of the four panels. In addition, there were no Grade 3 or 4 laboratory-related AEs that were considered related to any of the study drugs. Differences in AE incidence among patients who received ribavirin and those who did not in Panels 1 and 2 (Table 2) were a result of ribavirin-related adverse drug reactions, as were the respective differences observed in the incidence of laboratory abnormalities in Panels 1-3 (see Additional file 1). A comparable safety profile was observed with TMC647055/ ritonavir 450/30 $\mathrm{mg}$ and TMC647055/ritonavir 600/50 mg (Panels 1-3) and also between the JNJ-56914845 30-mg and 60-mg doses (Panel 4).

\section{Conclusion}

High SVR rates of 93\% and 100\% in GT1a- and GT1binfected patients, respectively, were achieved in this study by combining simeprevir $75 \mathrm{mg}$ with JNJ$5691484560 \mathrm{mg}$ and TMC647055/ritonavir 450/30 mg. Lower SVR rates were observed without the inclusion of JNJ-56914845. Based on the results of this study, the efficacy and safety of DAA-combination regimens targeting the same viral proteins as the drugs in this study (i.e., the HCV NS5B polymerase, the HCV NS5A protein and the $\mathrm{HCV}$ NS4/4A protease) are being investigated further.

\section{Additional files}

Additional file 1: Further information on the methods of the study, including dosing rationale and protocol deviations are included. In addition, further virologic resistance testing and safety results are included. (DOCX $16 \mathrm{~kb}$ )

Additional file 2: Figure S1. Mean (SD) plasma concentration versus time profile for: (a) simeprevir; (b) TMC647055; and (c) ritonavir in Panels 1-4; and for (d) JNJ-56914845 in Panel 4. RBV ribavirin, RTV ritonavir, SD standard deviation, SMV simeprevir. (DOCX $564 \mathrm{~kb}$ )

Additional file 3: Table S1. Week-4 JNJ-56914845 pharmacokinetic parameters after administration in Panel 4. (DOCX $14 \mathrm{~kb}$ ) 
Additional file 4: Table S2. Week-4 simeprevir pharmacokinetic parameters after administration in (a) Panels 1-3 and (b) Panel 4. (DOCX 15 kb)

Additional file 5: Table S3. Week-4 TMC647055 pharmacokinetic parameters after administration in (a) Panels $1-3$ and (b) Panel 4. (DOCX 15 kb)

Additional file 6: Table S4. Week-4 ritonavir pharmacokinetic parameters after administration in (a) Panels 1-3 and (b) Panel 4. (DOCX 15 kb)

\section{Abbreviations}

AE: Adverse event; $\mathrm{AUC}_{0-24 \mathrm{~h}}$ : Area under the plasma concentration-time curve over 24 h; BID: Twice daily; BQL: Below quantification limit; $C_{\text {max }}$ : Maximum plasma concentration; $C_{\text {min }}$ : Minimum plasma concentration CYP: Cytochrome P450; DAA: Direct-acting-antiviral agent; DET: Detectable; EOT: End of treatment; EU: European Union; FU: Follow-up; GT: Genotype; HCV: Hepatitis C virus; HIV: Human immunodeficiency virus; IFN: Interferon; ITT: Intent-to-treat; LDL: Low-density lipoprotein; MCHC: Mean corpuscular haemoglobin concentration; NNI: Non-nucleoside inhibitor; OATP: Organic anion transporting polypeptide; pegIFN: Pegylated interferon a-2a; PI: Protease inhibitor; RAV: Resistance-associated variant; RBV: Ribavirin; RTV: Ritonavir; SAE: Serious adverse event; SD: Standard deviation; SMV: Simeprevir; SVR: Sustained virologic response; SVR12: Sustained virologic response 12 weeks after end of treatment; SVR24: Sustained virologic response 24 weeks after end of treatment; VBT: Viral breakthrough WBC: White blood cell

\section{Acknowledgements}

The authors would like to thank the patients and investigators for their contributions to this study. Medical writing support was provided by Chrissie Kouremenou of Complete Medical Communications and funded by Janssen.

\section{Funding}

The study was funded by Janssen. Janssen were responsible for the study design and the collection and interpretation of data. Medical writing support was funded by Janssen.

\section{Availability of data and material}

The datasets generated during and/or analysed during the current study are available from the corresponding author on reasonable request.

\section{Authors' contributions}

$\mathrm{SB}, \mathrm{HW}, \mathrm{CM}, \mathrm{HO}, \mathrm{FN}, \mathrm{KA}, \mathrm{YH}, \mathrm{JMS}, \mathrm{PB}$ and SF were involved in the acquisition and interpretation of study data, and have been involved in the critical revision of the manuscript for important intellectual content. LV, TNK, EH, AV , $B J, P V R$ and RV were involved in the study concept and design, and the acquisition, analysis and interpretation of study data. They have also been involved in the critical revision of the manuscript for important intellectual content. DL provided statistical analysis of the study data, and has been involved in the critical revision of the manuscript for important intellectual content. All authors read and approved the final manuscript.

\section{Competing interests}

SB has received grants from Janssen, MSD and Roche, and has been on advisory boards for AbbVie, Bristol-Myers Squibb, Gilead Sciences, Janssen and MSD.

HW has received grants from Janssen.

CM has been an investigator for Roche, Schering-Plough, Gilead Sciences, Pfizer and Tibotec, has received research grants from Schering-Plough and Roche, has acted as an adviser/consultant for Janssen-Cilag and ScheringPlough, and as a speaker for Schering-Plough and Bristol-Myers Squibb. $\mathrm{HO}$ has acted as a consultant for Janssen (National Advisory Board) for the past 3 years.

FN has received research grants from Roche, Astelles, Ferring, Novartis, Janssen-Cilag and AbbVie, and has consultancy agreements with CAF, Intercept, Gore, Bristol-Myers Squibb, AbbVie, Novartis, MSD, Janssen-Cilag, Promethera, Ono Pharma and Durect.

KA has received advisory and consultancy fees from Boehringer Ingelheim, Bristol-Myers Squibb, Gilead, Hexal and Merck, and has been a clinical investigator for Bionor, Boehringer Ingelheim, Bristol-Myers Squibb, Gilead, GlaxoSmithKline, Janssen, Merck and Pfizer.
$\mathrm{YH}$ has received grants from Janssen, Merck and Roche, and has been a clinical investigator and/or consultant for Vertex, Janssen, Bristol-Myers Squibb, Merck, Roche, Gilead, AbbVie and Boehringer Ingelheim. JMS has received advisor and speaker fees from AbbVie, Bristol-Myers Squibb, Gilead, Janssen and Merck, and has been a clinical investigator for Boehringer Ingelheim, Gilead and Janssen.

PB has acted as a consultant for AbbVie, Bristol-Myers Squibb, Gilead, Janssen, Merz and MSD, and has given sponsored lectures for AbbVie, Bristol-Myers Squibb, Gilead, Falk, Janssen, Merck, Merz, MSD and Roche. SF has nothing to disclose.

LV, TNK, EH, DL, AV, BJ, PVR and RV are employees of Janssen and may own stock in Johnson \& Johnson.

\section{Consent for publication}

Not applicable.

\section{Ethics approval and consent to participate}

The study was approved by the Institutional Review Board or Independent Ethics Committee at each participating centre, and met the ethical principles of the Declaration of Helsinki and Good Clinical Practice guidelines. All patients provided written, informed consent

\section{Author details}

${ }^{1}$ Department of Gastroenterology \& Hepatology, ZNA Antwerp, Antwerpen, Belgium. ${ }^{2}$ Department of Hepatology and Gastroenterology, Universitair Ziekenhuis Gent, Ghent, Belgium. ${ }^{3}$ Liver Unit, CUB Hôpital Erasme, Université Libre de Bruxelles, Brussels, Belgium. ${ }^{4}$ Department of Gastroenterology and Hepatology, AZ Sint-Jan AV Hospital, Bruges, Belgium. ${ }^{5}$ Department of Liver and Biliopancreatic Disorders, University Hospitals KU Leuven, Leuven, Belgium. ${ }^{6}$ EPIMED/Vivantes-Auguste-Viktoria-Klinikum, Berlin, Germany. ${ }^{7}$ Hepato-gastroenterology, Cliniques Universitaires Saint-Luc, Université Catholique de Louvain, Brussels, Belgium. ${ }^{8}$ Department of Medicine, University Medical Center Mainz, Mainz, Germany. ${ }^{9} \mathrm{IFI}$, Liver Center Hamburg, Asklepiosklinik St. Georg, Hamburg, Germany. ${ }^{10}$ Department of Gastroenterology and Hepatology, Universitair Ziekenhuis Antwerpen, Antwerp, Belgium. ${ }^{11}$ Janssen Pharmaceutica NV, Beerse, Belgium. ${ }^{12}$ Alios BioPharma Inc, South San Francisco, CA, USA. ${ }^{13}$ Janssen Research \& Development BVBA, Beerse, Belgium. ${ }^{14}$ Janssen Pharmaceuticals LLC, Titusville, NJ, USA.

Received: 6 October 2016 Accepted: 18 January 2017

Published online: 10 February 2017

\section{References}

1. Florian J, Mishra P, Arya V, Harrington P, Connelly S, Reynolds KS, et al. Direct-acting antiviral drugs for the treatment of chronic hepatitis $C$ virus infection: interferon free is now. Clin Pharmacol Ther. 2015;98:394-402.

2. Ferenci P, Bernstein D, Lalezari J, Cohen D, Luo Y, Cooper C, et al. ABT-450/ r-ombitasvir and dasabuvir with or without ribavirin for HCV. N Engl J Med. 2014;370:1983-92.

3. Sanford M. Simeprevir: a review of its use in patients with chronic hepatitis C virus infection. Drugs. 2015;75:183-96.

4. Ouwerkerk-Mahadevan S, Snoeys J, Peeters M, Beumont-Mauviel M, Simion A. Drug-drug interactions with the NS3/4A protease inhibitor simeprevir. Clin Pharmacokinet. 2016:55:197-208.

5. Cummings MD, Lin Tl, Hu L, Tahri A, McGowan D, Amssoms K, et al. Discovery and early development of TMC647055, a non-nucleoside inhibitor of the hepatitis C virus NS5B polymerase. J Med Chem. 2014;57:1880-92.

6. Bourgeois S, Reesink HW, Leempoels J, Vijgen L, Rouan MC, Marien K, et al. Combination therapy of TMC647055 with simeprevir (TMC435) in chronic hepatitis C patients [abstract]. J Hepatol. 2013;58:S483.

7. Gardner S, Cutrell A, Elko-Simms C, Adkison K, Hamatake R, Walker J, et al. A double-blind, randomized, placebo-controlled study to assess the safety, antiviral activity and pharmacokinetics of GSK2336805 when given as monotherapy and in combination with peginterferon alfa-2a and ribavirin in hepatitis C virus genotype 1-infected treatment-naive subjects. Liver Int. 2014;34:e89-95.

8. Walker J, Crosby R, Wang A, Woldu E, Vamathevan J, Voitenleitner C, et al. Preclinical characterization of GSK2336805, a novel inhibitor of hepatitis C virus replication that selects for resistance in NS5A. Antimicrob Agents Chemother. 2014;58:38-47. 
9. Vanwelkenhuysen I, de Vries R, Timmerman P, Verhaeghe T. Determination of simeprevir: a novel, hepatitis $C$ protease inhibitor in human plasma by high-performance liquid chromatography-tandem mass spectrometry. J Chromatogr B Analyt Technol Biomed Life Sci. 2014;958:43-7.

10. Andreone P, Colombo MG, Enejosa JV, Koksal I, Ferenci P, Maieron A, et al. ABT-450, ritonavir, ombitasvir, and dasabuvir achieves 97\% and $100 \%$ sustained virologic response with or without ribavirin in treatmentexperienced patients with HCV genotype $1 \mathrm{~b}$ infection. Gastroenterology. 2014;147:359-65.

11. Feld JJ, Kowdley KV, Coakley E, Sigal S, Nelson DR, Crawford D, et al. Treatment of HCV with ABT-450/r-ombitasvir and dasabuvir with ribavirin. N Engl J Med. 2014;370:1594-603.

12. Zeuzem S, Jacobson IM, Baykal T, Marinho RT, Poordad F, Bourlière $M$, et al. Retreatment of HCV with ABT-450/r-ombitasvir and dasabuvir with ribavirin. N Engl J Med. 2014:370:1604-14.

13. Forns $X$, Lawitz E, Zeuzem S, Gane E, Bronowicki JP, Andreone P, et al. Simeprevir with peginterferon and ribavirin leads to high rates of SVR in patients with HCV genotype 1 who relapsed after previous therapy: a phase 3 trial. Gastroenterology. 2014;146:1669-79.

14. Jacobson IM, Dore GJ, Foster GR, Fried MW, Radu M, Rafalsky W, et al. Simeprevir with pegylated interferon alfa 2a plus ribavirin in treatment-naive patients with chronic hepatitis C virus genotype 1 infection (QUEST-1): a phase 3, randomised, double-blind, placebo-controlled trial. Lancet. 2014;384:403-13.

15. Manns M, Marcellin P, Poordad F, de Araujo ES, Buti M, Horsmans $Y$, et al. Simeprevir with pegylated interferon alfa $2 \mathrm{a}$ or $2 \mathrm{~b}$ plus ribavirin in treatment-naive patients with chronic hepatitis $C$ virus genotype 1 infection (QUEST-2): a randomised, double-blind, placebo-controlled phase 3 trial. Lancet. 2014;384:414-26.

16. Kakuda T, Spittaels K, Verloes R, Vanwelkenhuysen I, Truyers C, Van Remoortere P. Pharmacokinetics of simeprevir, JNJ-56914845 and ritonavir-boosted TMC647055 when co-administered in healthy volunteers. 2014. http://regist2. virology-education.com/abstractbook/2014_4.pdf. Accessed 23 Aug 2016.

\section{Submit your next manuscript to BioMed Central and we will help you at every step:}

- We accept pre-submission inquiries

- Our selector tool helps you to find the most relevant journal

- We provide round the clock customer support

- Convenient online submission

- Thorough peer review

- Inclusion in PubMed and all major indexing services

- Maximum visibility for your research

Submit your manuscript at www.biomedcentral.com/submit 